$01.5 ; 09.5$

\title{
Влияние структуры волнового фронта лазерного излучения на синхронные автоколебания в волоконных лазерах с микрооптомеханическими резонаторами
}

\author{
(C) Ф.А. Егоров
}

Фрязинский филиал Института радиотехники и электроники им. В.А. Котельникова РАН, Фрязино, Московская обл., Россия

E-mail: egorov-fedor@mail.ru

Поступило в Редакцию 22 октября 2021 г.

В окончательной редакции 31 января 2022 г.

Принято к публикации 1 февраля 2022 г.

\begin{abstract}
Показана возможность повышения стабильности и устойчивости синхронных автоколебаний в волоконных лазерах с нелинейными зеркалами на основе микрооптомеханических резонаторов (микроосцилляторов) за счет трансформации структуры волнового фронта лазерного излучения, взаимодействующего с микроосцилляторами. В основе указанных преобразований лежат уникальные свойства многомодовой интерференции в составных волоконных световодах типа singlemode-multimode и singlemode-multimode-singlemode.
\end{abstract}

Ключевые слова: синхронные автоколебания, волоконный лазер, микрокантилевер, резонанс.

DOI: 10.21883/PJTF.2022.07.52292.19060

Микроосцилляторы (MOMR) позволяют реализовать в лазерах новые интересные режимы работы с уникальными характеристиками генерируемого излучения [1-4]. В волоконных лазерах (FL) с микроосцилляторами благодаря лазерному возбуждению упругих колебаний в MOMR можно получить режимы синхронных автоколебаний (CA) с модуляцией параметров генерируемого излучения на частотах, близких к собственным частотам $(f)$ мод упругих колебаний MOMR: $F=(1+\chi) f$, $\chi \ll 1$ [5]. Это может найти применение в волоконных лазерных источниках импульсного излучения для стабилизации частоты импульсов (аналог „кварцевойб стабилизации в радиотехнике), а также может служить основой для разработки нового класса резонансных волоконно-оптических датчиков [6,7].

Динамика лазерных систем FL-MOMR существенно зависит от условий взаимодействия лазерного излучения с MOMR, определяющих, в частности, эффективность возбуждения упругих колебаний, характер и глубину модуляции световой волны. При этом отметим, что влияние такого важного фактора, как структура волнового поля, на взаимодействие излучения с упругими колебаниями MOMR и автоколебания в FL-MOMR изучено явно недостаточно. Структурой волнового фронта лазерного (когерентного) излучения в оптоволоконных системах можно эффективно управлять за счет использования многомодовой интерференции в составных волокнах вида singlemode-multimode (SM) и singlemode-multimode-singlemode (SMS) [8,9], что обусловливает актуальность исследований лазерных систем $\mathrm{FL}-\mathrm{SM}(\mathrm{S})-\mathrm{MOMR}$.

Эксперименты выполнены с помощью эрбий-иттербиевого волоконного лазера (EYDFL) с диодной накачкой, в котором микроосциллятор играет роль зер- кала $(M)$ лазерного резонатора (рис. 1). Накачка активного волокна (AF) с двойной оболочкой производится непрерывным излучением диода накачки $\left(\lambda_{p} \approx 0.98 \mu \mathrm{m}\right)$, направляемым во внутреннюю оболочку $\mathrm{AF}\left(d_{c l} \approx 105 \mu \mathrm{m}\right)$ с помощью многомодового волоконного разветвителя, который используется также для регистрации излучения, генерируемого EYDFL $\left(\lambda_{s} \approx 1540 \mathrm{~nm}\right)$. Одномодовая сердцевина $\mathrm{AF}$ с диаметром $d_{c o} \approx 9 \mu \mathrm{m}$ легирована эрбием и иттербием с концентрациями $\left(N_{\mathrm{Er}} ; N_{\mathrm{Yb}}\right) \approx\left(5 \cdot 10^{24} ; 1.5 \cdot 10^{26} \mathrm{~m}^{-3}\right)$, длины AF и волоконно-оптического резонатора EYDFL $\left(L_{\mathrm{AF}} ; L\right) \approx(0.9 ; 1 \mathrm{~m})$, коэффициенты отражения полупрозрачного дихроичного зеркала $\left(M_{0}\right) r_{0}\left(\lambda_{s}\right) \approx 95 \%$, $r_{0}\left(\lambda_{p}\right)<6 \%$, ширина огибающей одномодового многочастотного лазерного излучения $\Delta \lambda_{s} \lesssim 1 \mathrm{~nm}$. Средняя мощность лазерного излучения, падающего на MOMR, могла варьироваться в пределах $\bar{P}_{s}=0-8 \mathrm{~mW}$ за счет регулировки мощности накачки $\left(P_{p}\right)$.

В экспериментах использованы кремниевые MOMR c колебательными элементами в виде микрокантилеверов (МC), различающихся параметрами мод упругих колебаний, оптическими свойствами, размерами: $\mathrm{MC}_{1}, \mathrm{MC}_{2}$. Поверхность $\mathrm{MC}_{1}$, взаимодействующая $\mathrm{c}$ лазерным излучением, покрыта тонкой пленкой никеля $(\sim 120 \mathrm{~nm})$ с коэффициентами отражения и поглощения $R_{1}\left(\lambda_{s}\right) \approx 70 \%, A_{1}\left(\lambda_{s}\right) \approx 30 \%$, а рабочая поверхность $\mathrm{MC}_{2}$ покрыта многослойной интерференционной пленкой $\mathrm{ZrO}_{2}+\mathrm{SiO}_{2}$ с высоким отражением $R_{2}\left(\lambda_{s}\right) \approx 98 \%$, $A_{2}\left(\lambda_{s}\right)<0.1 \%$. Различие оптических свойств $\mathrm{MC}_{1,2}$ приводит к существенно разным механизмам лазерного возбуждения в них изгибных колебаний $U(z, t)[10]$ : в $\mathrm{MC}_{1}$ реализуется в основном фототермическое возбуждение, обусловленное поглощением излучения в $\mathrm{Ni}$ пленке, в $\mathrm{MC}_{2}$ - за счет давления света. Заданное 


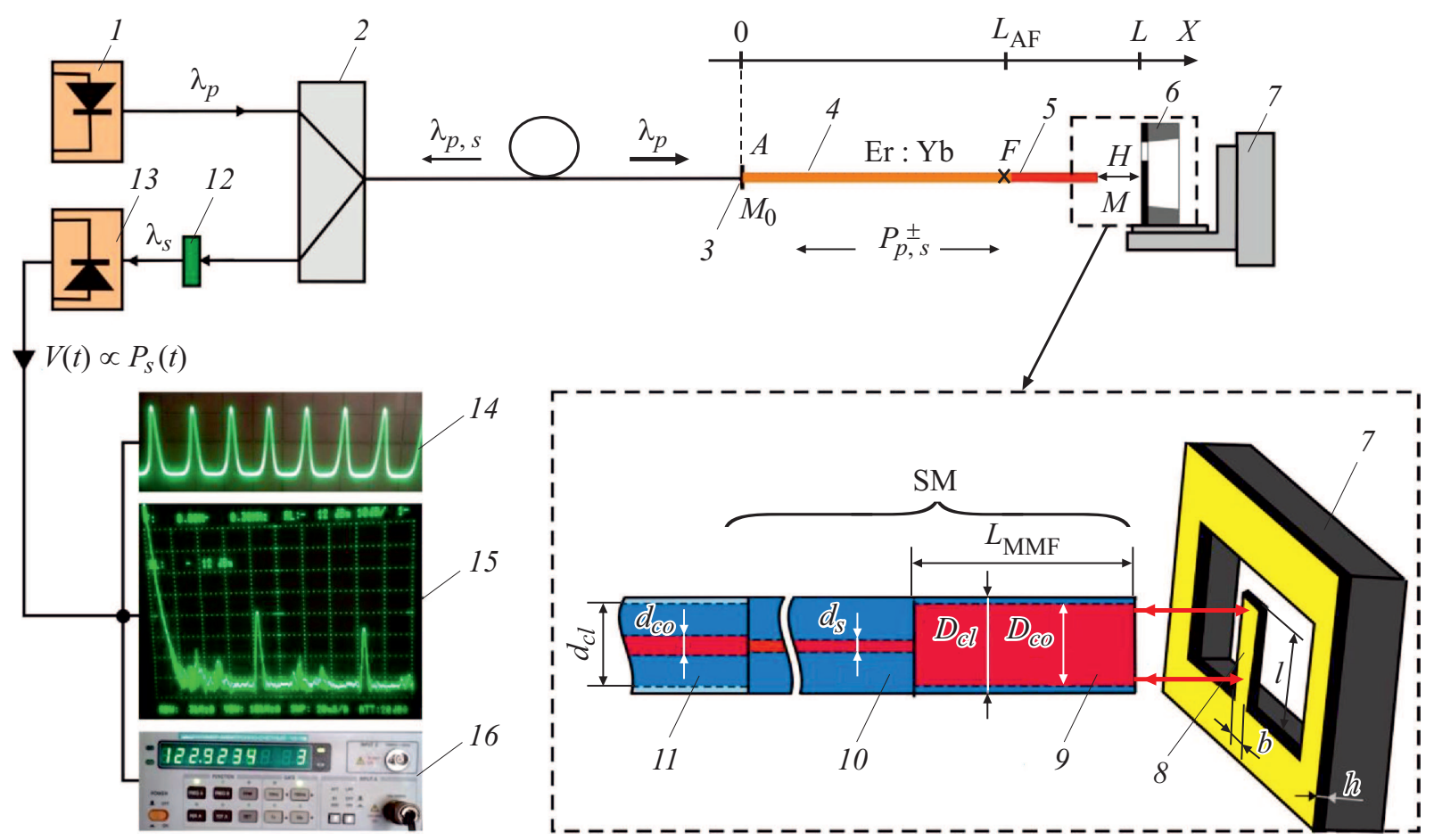

Рис. 1. Схема лазерной системы EYDFL-SM-MOMR. 1 - полупроводниковый лазер накачки $\left(\lambda_{p} \approx 980 \mathrm{~nm}\right), 2-$ волоконнооптический разветвитель (многомодовый), 3 - дихроичное зеркало $\left(M_{0}\right), 4$ - эрбий-иттербиевый активный световод с двойной оболочкой, 5 - SM, 6 - корпус MOMR, 7 - микропозиционер $(x-y-z), 8-$ микрозеркало (M)-кремниевый микрокантилевер $\left(\mathrm{MC}_{1}: 520 \times 75 \times 24 \mu \mathrm{m}, \mathrm{MC}_{2}: 660 \times 73 \times 6 \mu \mathrm{m}\right), 9$ - многомодовый волоконный ступенчатый световод (MMF), 10 - одномодовый волоконный световод (SMF), $11-\mathrm{AF}, 12$ - оптический фильтр, 13 - фотоприемник (выходной сигнал $\left.V(t) \propto P_{s}(t)\right), 14$ - осциллограф (Tektronix 2465), 15 - анализатор спектра радиочастот (Anritsu MS 710C), 16 частотомер (Ч3-163). На экранах осциллографа и анализатора спектра показаны форма импульсов и фурье-спектр интенсивности лазерного излучения в $\mathrm{EYDFL}-\mathrm{SM}_{2}-\mathrm{MC}_{1}$ при возбуждении CA на собственной частоте второй моды поперечных колебаний $\mathrm{MC}$. Волоконные световоды AF, SM и MMF соединены с помощью сварки.

положение SM (выходного торца МMF) относительно $\mathrm{MC}_{1,2}$ обеспечивалось с помощью трехкоординатного микропозиционера.

Схема SM показана на рис. 1. Вместо упрощенных $\mathrm{SM}$, полученных путем соединения AF непосредственно с MMF, нами разработаны SM с „переходным“ $\mathrm{SMF}$, что позволяет улучшить свойства SM за счет вариации параметров переходных SMF и устранить остаточное излучение накачки, проходящее из AF в MMF и возмущающее колебания микроосциллятора. Доля мощности излучения, обратно отраженного от MOMR в AF: $R_{e f f}(t)=R_{1,2} T_{s} K(t)=\bar{R}_{e f f}+r(t)$, где $K(t)$ - эффективный коэффициент оптической связи $\mathrm{SM}$ c MOMR, зависящий от колебаний $\mathrm{MC}_{1,2} ; T_{s}-$ передаточная функция SMS с удвоенной длиной MMF; $\bar{R}_{e f f}, r(t)$ - постоянная и переменная составляющие. Были изготовлены SM с разными типами кварцевых $\mathrm{SMF}$ и MMF, при этом основная часть экспериментов выполнена с помощью SM на основе ступенчатого MMF с размерами $D_{c o} / D_{c l}=105 / 125 \mu \mathrm{m}$, числовой апертурой $N A_{M}=\left(n_{c o}^{2}-n_{c l}^{2}\right)^{1 / 2} \approx 0.22$ и SMF (G657A2): $N A_{S} \approx 0.16, d_{s} \approx 6 \mu \mathrm{m}$, приводящих к типичным значениям $\bar{R}_{\text {eff }}=0.2-0.5$.
При $N A_{S, M} \ll 1$ свойства SM определяются в основном параметрами MMF-секции [8]. Так, ключевая характеристика SM - пространственный период $\left(L_{0}\right)$ „изображений“ источника когерентного излучения, возникающих в MMF в результате многомодовой интерференции, - выражается в виде $L_{0}=4 n_{c o} D_{c o}^{2} / \lambda_{s}$, при этом структура ближнего поля в выходном сечении ММF зависит от соотношения $k=L_{\mathrm{MMF}} / L_{0}$. С учетом $n_{c o}\left(\lambda_{s}\right) \approx 1.456$ для рассматриваемых $\mathrm{SM}$ $L_{0} \approx 41.62 \mathrm{~mm}$. Мы ограничимся рассмотрением трех типов $\mathrm{SM}_{1-3}$, имеющих $k_{1-3}=1,1 / 2,0.584$. Для случая $k_{2}=1 / 2$, представляющего особый интерес, на рис. $2, a, b$ показано радиальное распределение интенсивности $\left(I_{s}\right)$ лазерного излучения в выходном сечении $\mathrm{MMF}$, которое получено путем измерения оптической мощности, поступающей в приемный SMF (G657A2) через малый зазор $(\lesssim 5 \mu \mathrm{m})$ из разных участков торцевой поверхности ММF. При $k_{1}=1$ излучение практически полностью локализовано в центре выходного сечения MMF в виде яркого пятна с диаметром $\sim d_{s}$, которое по существу является „изображением“ модового пятна $\mathrm{SMF}$. При $L_{\mathrm{MMF}}=L_{0} / 2$ волновой фронт излучения имеет форму тонкого кольца с шириной $\sim d_{s}$, расположен- 


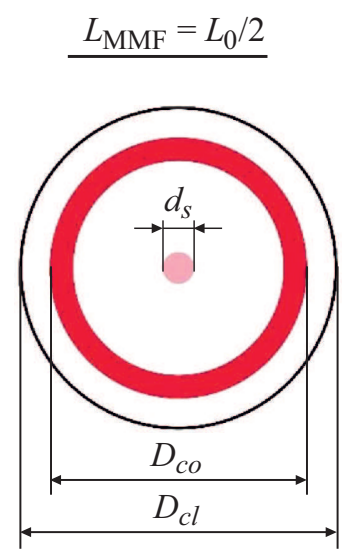

$a$

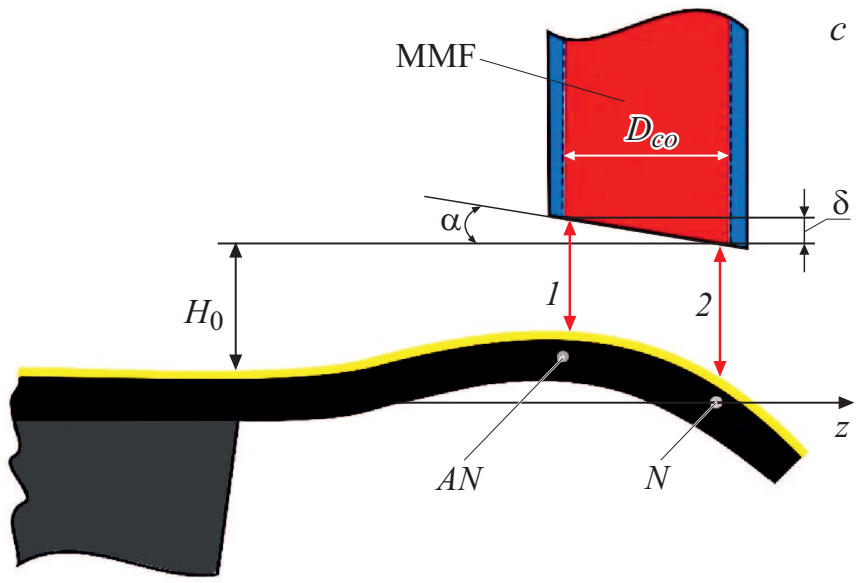

$b$

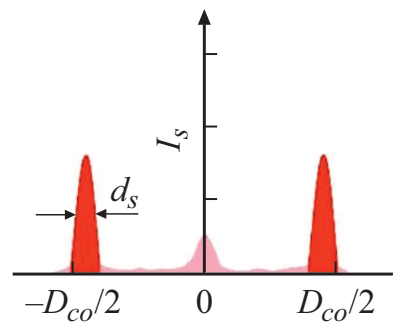

$d$

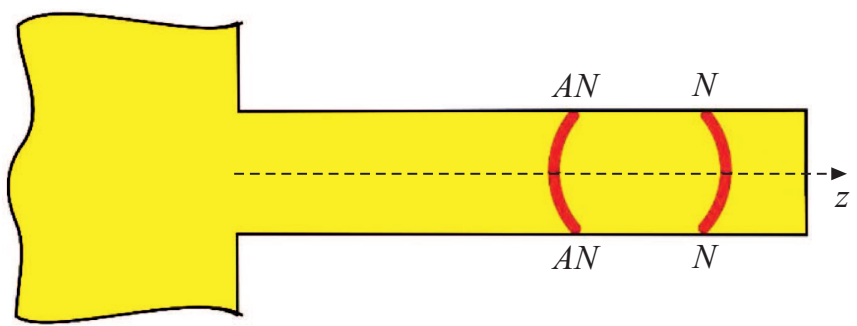

Рис. 2. Схема $(a)$ и диаграмма $(b)$ радиального распределения интенсивности лазерного излучения в ближней зоне выходного сечения MMF при $k_{2}=1 / 2 ; c-$ схема взаимодействия MOMR с лазерным излучением с кольцеобразным волновым фронтом в условиях возбуждения второй моды поперечных колебаний $\mathrm{MC}_{1} ; d-$ проекция лазерного пучка на $\mathrm{MC}_{1}$.

ного вдоль периметра сердцевины МMF, при этом доля мощности в слабом центральном пятне $\lesssim 5 \%$ от полной мощности в $\mathrm{MMF}\left(P_{s}\right)$. В случае $k_{3} \approx 0.584$ излучающая область торцевой поверхности МMF имеет вид концентрической системы с ярким пятном в центре и узким кольцом вдоль периметра сердцевины MMF, которые обладают примерно равными мощностями $\left(\sim P_{s} / 2\right)$. В указанных случаях реализуются существенно разные условия взаимодействия лазерного излучения с MOMR.

Установлено, что в исследованных лазерных системах $\mathrm{EYDFL}-\mathrm{SM}_{1-3}-\mathrm{MC}_{1,2}$ в условиях резонанса вида $f_{\text {rel }} \approx f\left(f_{\text {rel }}\left(P_{p}\right)\right.$ - частота релаксационных колебаний в $\mathrm{FL}) \mathrm{CA}$ с частотами $F \approx f$ существуют независимо от особенностей механизмов лазерного возбуждения колебаний, оптических свойств и конструкции МС. Реализованы условия резонанса и СА (в частности, за счет регулировки мощности накачки) на первой, второй модах изгибных колебаний $\mathrm{MC}_{1} \mathrm{c}$ собственными частотами $\left(f_{1,1} ; f_{1,2}\right)=(22.4 ; 122.9 \mathrm{kHz})$ и первой, второй, третьей модах $\mathrm{MC}_{2}$ с частотами $\left(f_{2,1} ; f_{2,2} ; f_{2,3}\right)=(6.9 ; 38.7 ; 118.1 \mathrm{kHz})$, имеющих механические добротности (на воздухе) $Q_{1}=40-90$, $Q_{2}=30-110$. При $k_{1}=1$ ввиду идентичности характеристик излучения источника и его „изображения“ (на выходе MMF) свойства лазерных систем EYDFL-SM $-\mathrm{MC}_{1,2}$ и EYDFL-MC $\mathrm{MC}_{1,2}$ практически совпадают. В них СА обусловлены модуляцией
$R_{e f f}(t)$ при колебаниях базы резонатора Фабри-Перо $\left(H(t)=H_{0}+U(t)\right)$, образованного полуотражающей ( $4 \%$ ) торцевой поверхностью MМF (либо SMF) и отражающей колеблющейся поверхностью МС (исходная база $\left.H_{0} \lesssim 20 \mu \mathrm{m}\right)$. В них ввиду периодичности $R_{e f f}(H)$ изменения базы $\Delta H_{0} \geqslant \lambda_{s} / 2$ приводят к сменам знака обратной связи и тем самым к срывам СА.

$\mathrm{B} \mathrm{SM} 2,3$ из-за сложной (распределенной) формы волнового фронта на выходе MMF, обратноотраженный в $\mathrm{AF}$ сигнал формируется в результате интерференции нескольких пучков, отраженных от разных участков поверхности МC (рис. 2, $c, d$ ), испытывающих разные деформации при колебаниях. С точки зрения существования СА здесь отражение от торцевой поверхности MMF $(\sim 4 \%)$ не играет важной роли (в отличие от схем с $\left.\mathrm{SM}_{1}\right)$, поскольку как поступательное смещение MOMR, изменяющее зазор $\Delta H_{0} \geqslant \lambda_{s} / 2$, так и просветление торцевой поверхности MMF практически не влияли на СА. Это объясняется тем, что в данном случае модуляция $R_{e f f}$, вызывающая СА, зависит в основном от разности фаз интерферирующих лучей, так что общие для них приращения оптических длин, обусловленные перемещением MOMR, компенсируются, а „фоновое“ отражение от торца MMF ( 4\%) практически не влияет на модулированную составляющую $r(t)$. Таким образом, в FL-MOMR трансформация волнового фронта с по- 
мощью $\mathrm{SM}_{2,3}$ приводит к повышению стабильности и устойчивости СА.

Наряду с этим указанные формы волновых фронтов в $\mathrm{SM}_{2,3}$ позволяют реализовать в $\mathrm{FL}-\mathrm{MOMR}$ условия, обеспечивающие высокую эффективность как для лазерного возбуждения колебаний МC, так и для модуляции излучения при ее колебаниях - ключевых факторов СА. Заметим, что координаты областей фокусировки излучения на МС, обеспечивающие по отдельности максимумы для амплитуд колебаний МС и глубины модуляции излучения, определяются существенно разными условиями и, как правило, не совпадают. Эти условия зависят, например, от моды и механизма возбуждения упругих колебаний [10], модулируемого параметра световой волны (амплитуды, фазы и др.). В рассматриваемых лазерных системах возможность оптимизации взаимодействия микроосциллятора с лазерным излучением связана с тем, что одна часть волнового фронта может быть локализована в области эффективного возбуждения колебаний, а другая часть - в области максимальной глубины модуляции, что в итоге приводит к повышению результирующего эффекта от обоих факторов. Такой подход применим, например, при возбуждении высоких мод колебаний МС, имеющих пучности $(A N)$ и узлы - неподвижные точки $(N)$, при этом одну часть волнового фронта целесообразно расположить вблизи узла, а другую - в пучности колебаний. Это подтверждается, в частности, результатами исследований $\mathrm{CA}$, возбуждаемых в EYDFL- $\mathrm{SM}_{2}-\mathrm{MC}_{1}$ на частоте $F_{2} \approx 122.9 \mathrm{kHz}$ второй моды колебаний $\mathrm{MC}_{1}$ (рис. 1 и $2, c, d)$, где излучение, падающее в область пучности, приводит к эффективному возбуждению колебаний, тогда как интерференция лучей 1,2 , отраженных в зонах пучности и узла, приводит к эффективной модуляции $R_{e f f}(t)$. При этом условие „квадратуры“ для данных лучей реализовано за счет шлифовки торца MMF под малым углом $\alpha=\delta / D_{c o} \approx 0.12^{\circ}$, обеспечивающим исходный оптимальный сдвиг фаз между лучами 1, 2: $2 \delta\left(n_{c o}-1\right)=\lambda_{s} / 8$. В итоге СА в данной лазерной системе на частоте второй моды колебаний $\mathrm{MC}_{1}$ обладали кратковременной стабильностью частоты (периода) $\left|\Delta F_{2} / F_{2}\right|_{f l} \approx 6 \cdot 10^{-6}$ (время усреднения частотомера $0.1 \mathrm{~s}$ ), при этом „долговременная“ нестабильность частоты СА при нормальных условиях по данным двухмесячных непрерывных наблюдений (без срывов СА) не превышала $\left|\Delta F_{2} / F_{2}\right| \approx 10^{-5}$. Полагаем, что $\mathrm{SM}_{3}$ целесообразно использовать в $\mathrm{FL}-\mathrm{MOMR}$ при возбуждении CA, например, на аксиально симметричных модах упругих колебаний MOMR, в частности микромембран.

Таким образом, в волоконных лазерах с микроосцилляторами преобразование структуры волнового фронта лазерного излучения, взаимодействующего с модами упругих колебаний, позволяет повысить устойчивость и долговременную стабильность СА, при этом с ростом гармоник возбуждаемых мод независимо от механизмов лазерного возбуждения упругих колебаний значительно расширяются возможности для оптимизации взаимодействия, приводящей к указанным положительным эффектам.

\section{Финансирование работы}

Работа выполнена в рамках госзадания ИРЭ им. В.А. Котельникова РАН.

\section{Конфликт интересов}

Автор заявляет, что у него нет конфликта интересов.

\section{Список литературы}

[1] W. Yang, S.A. Gerke, K.W. Ng, Y. Rao, C. Chase, C.J. ChanHasnain, Sci. Rep., 5, 13700 (2015). DOI: 10.1038/srep13700

[2] M. Fabert, A. Desfarges-Berthelmot, V. Kermene, Opt. Express, 20, 22895 (2012). DOI: 10.1364/OE.20.022895

[3] D. Princepe, G.S. Wiederhecker, I. Favero, N.C. Frateschi, IEEE Photon. J., 10, 4500610 (2018). DOI: 10.1109/JPHOT.2018.2831001

[4] X. Xiang, M. Jingwen, S. Xiankai, Phys. Rev. A, 99, 053837 (2019). DOI: 10.1103/PhysRevA.99.053837

[5] Ф.А. Егоров, В.Т. Потапов, Квантовая электроника, 50 (8), 734 (2020). [F.A. Egorov, V.T. Potapov, Quantum Electronics, 50 (8), 734 (2020). DOI: 10.1070/QEL17116].

[6] Ф.А. Егоров, В.Т. Потапов, Фотон-Экспресс, № 7, 4 (2018). http://fotonexpres.ru/bez-rubriki/vyshel-iz-pechatifoton-ekspress-7

[7] E. Buks, I. Martin, Phys. Rev. E, 100, 032202 (2019). DOI: $10.1103 /$ PhysRevE.100.032202

[8] X. Zhu, A. Schulzgen, H. Li, L. Li, L. Han, J.V. Moloney, N. Peyghambarian, Opt. Express, 16, 632 (2008). DOI: $10.1364 / \mathrm{OE} .16 .016632$

[9] G. Bawa, K. Dandapat, G. Kumar, I. Kumar, S.M. Tripathi, IEEE Sensors J., 19, 6756 (2019). DOI: $10.1109 /$ JSEN.2019.2913801

[10] D. Ma, J.L. Garrett, J.N. Munday, Appl. Phys. Lett., 106, 091107 (2015). DOI: 10.1063/1.4914003 